\title{
Application of methods of the theory of multiphase queueing systems to the problems of optimizing the resources of service companies in the field of housing sector
}

\author{
Rustam Khayrullin ${ }^{1, *}$, and Alexey Myasnikov ${ }^{1}$ \\ ${ }^{1}$ Moscow State University of Civil Engineering, Yaroslavskoe shosse, 26, Moscow, 129337, Russia
}

\begin{abstract}
The scientific - methodological approach to calculation of duration and estimation of complexity of the expertise of technical and operational documentation for measuring equipment and technical devices used in the field of construction and housing sector is suggested. The approach is based on both the theory of queueing systems and probabilistic and statistical methods. The developed approach is implemented in the form of package of applied programs. The software based on complex using the principle of decomposition of object into constituent parts, the queuing theory and the method of expert estimations. The software allows on the basis of the results of statistical data processing for the past periods of work to receive both estimates of the average time of the expertise as a whole, and the estimates of the individual expertise stages. The algorithm for calculating the required number of employees for quality customer service is developed, taking into account the restriction on the waiting time of the request in the queue. The obtained results make it possible to make optimal use of working personnel at various work sites, taking into account their qualifications and training. The developed approach can be used to form a "fair price list" for private clients and enterprises - customers of expertise.
\end{abstract}

\section{Introduction}

The problem of planning labor costs and working time of scientific and technical staff during the scientific-technical works, as a whole, and its individual stages, seems relevant in many fields of activities. In [1] there describes developed a scientific and methodological approach to the assessment of the complexity of providing scientific and technical services in the field of metrology. This approach is based on the method of expert evaluations [2], which, as you know, has a certain level of subjectivity. Subjectivity of the results obtained by the method of expert assessments is due to the fact that various experts (expert groups) can be involved for expertise, and the opinions of experts may differ greatly from each other.

* Corresponding author: zrk@nm.ru 
In this paper, under the term expertise, we mean the examination of technical and operational documentation for measuring equipment (ME). The approach to the calculation of the duration of the expertise is based on the theory of queueing systems and probabilistic - statistical methods $[3,4]$. These methods are more stringent, so there is reason to believe that the results will be more reliable.

Currently, a wide range of products and a large number of ME and technical devices are used in the sphere of construction and housing and communal services. The main document establishing the nomenclature of $\mathrm{ME}$, that are meeting the modern technical requirements and using in technical systems, is a restrictive list of measuring equipment (RLME) [1].

The RMLE is periodically updated with the subsequent approval of the new edition of this document. Statistical data processing showed that the ME nomenclature included in the RMLE has a certain tendency to decrease $[1,2]$. In accordance with the current regulations, the use of ME absent in RMLE is allowed in technically justified cases in the presence of a positive reasoned conclusion (RC) issued by accredited organizations after the metrological expertise in accordance with the procedure established by methodological guidelines and regulatory documents. In case of a positive result of the expertise a $\mathrm{RC}$ is given on the possibility of using a sample of ME for assembling technical systems.

In this article, we outline the problem of estimating the labor costs needed to conduct the expertise of ME. The increase in the scope of work on the metrological expertise of (ME) makes the theme of articles relevant and practically meaningful.

\section{The decomposition of the problem}

Customers of the metrological expertise are private clients and medium or large enterprises. The process of customer service is modeled as the process of passing their requests (or applications) on expertise through a multiphase queueing system.

In accordance with the principle of decomposition, we divide the process of service of applications into the following stages:

1) Primary check of completeness of the documents provided by the customer;

2) Additional request for more information (ARMI) from the customer. Performed in the absence of all necessary documents and information in the initial request and submitted by the customer package of documents;

3) Repeated check of completeness of the submitted documents;

4) Carrying out preliminary expertise (includes, among other things, the analysis and determination of the complexity of the received application for expertise, depending on the number of $\mathrm{ME}$ in the application, the purpose of $\mathrm{ME}$ and measured physical quantities, the presence of software in $\mathrm{ME}$, etc.). Depending on the revealed complexity the corresponding application belongs to a certain group of complexity. The duration of the preliminary expertise depends significantly on the complexity group;

5) Request from the customer ARMI (performed in the event of insufficient necessary information identified at the stage of preliminary expertise);

6) Formation of pre-trial RC ("positive" or "negative");

7) Request from the customer ARMI. It is carried out in the case of pre-trial negative $\mathrm{RC}$ in the case where there is a possibility of issuing a positive RC, provided that the ARMI presented earlier is clarified, detailed and specified. This possibility can only be identified during the preparation of pre-trial RC.

8) The formation and signing of the positive RC;

9) Registration and signing of the agreement (contract) of rendering scientific and technical services in carrying out expertise. Payment for services under the contract. Issuance to the customer RC. 
Stages of works 1), 3), 6) were considered as single-phase queueing systems with waiting and unlimited queue. Incoming applications may contain from one to ten, and even fifteen ME. The complexity of such applications may vary significantly. In this regard, for the simulation of stage 4) were used several parallel connected single-phase queueing systems with varying service rate.

Stage 9) was deleted from the review, as this phase is performed by the staff of the Contract Department and the Financial - Accounting Department. It does not require the involvement the scientific - technical workers to providing the expertise.

The model took into account that at different stages of providing services to the customer after the request from the customer ARMI part of the applications may be dropped out of the system (the customer refuses or can not provide the requested ARMI and, therefore, finish interacting and refuse about expertise).

The total time from the moment of receipt of the application for expertise to the moment of issue of the RC includes duration of "direct" scientific - technical work with the application (duration of service of the application by system and duration of the queues arising if "the all servicing phases" is occupied) and duration of waiting of ARMI from the customer.

The value of probabilities of transition from one stage of work to another, as well as the value probabilities of disposal of applications at different stages of the expertise is assumed to be given. They are calculated before the mathematical simulation starts by means of statistical processing of historical data.

\section{The model of the multiphase queueing system}

Figure 1 presents the scheme of the multiphase system to serve applications from the three groups of difficulty. After the name of the expertise stage, the capacity of the corresponding single-phase queueing system is indicated.

As an input stream there considered the simplest Poison stream. The service time is assumed to be exponential (in fact, these assumptions are only approximate). Under these assumptions, it is possible to obtain the most significant and meaningful results of the analysis. In all other cases, the structure of outgoing single-phase queueing systems, which are incoming flows for other single-phase queueing systems, has a more complex probabilistic nature.

Let us denote

$\lambda$ - the intensity of the input flow;

$\mu$ - rate of flow service (capacity of service of queuieng system).

Then the stationary processes in system will be described by the standard system of algebraic equations [3]. The main characteristics of queueing system are [3]:

$\rho=\lambda / \mu$ - given the intensity (density) of the stream, $\rho<1$;

$P_{0}=1-\rho$ - probability that the service channel is free, there is no queue;

$P_{k}=\rho^{k} P_{0}$ - probability that the service channel and $k$ places in the queue are occupied;

$q=1$ - relative throughput of the system;

$A=q \lambda-$ absolute throughput of the system;

$M[r]=\rho^{2} /(1-\rho)$ - average number of applications in queue;

$M[s]=M[r]+1 / \mu$ - average number of requests in the queueing system; 
$T_{\text {que }}=M[r] / \lambda$ - average duration of stay orders in the queue;

$T_{s y s}=\left(\rho^{2} /(1-\rho)+1 / \mu\right) / \lambda$ - average time of application stay in the queueing system.

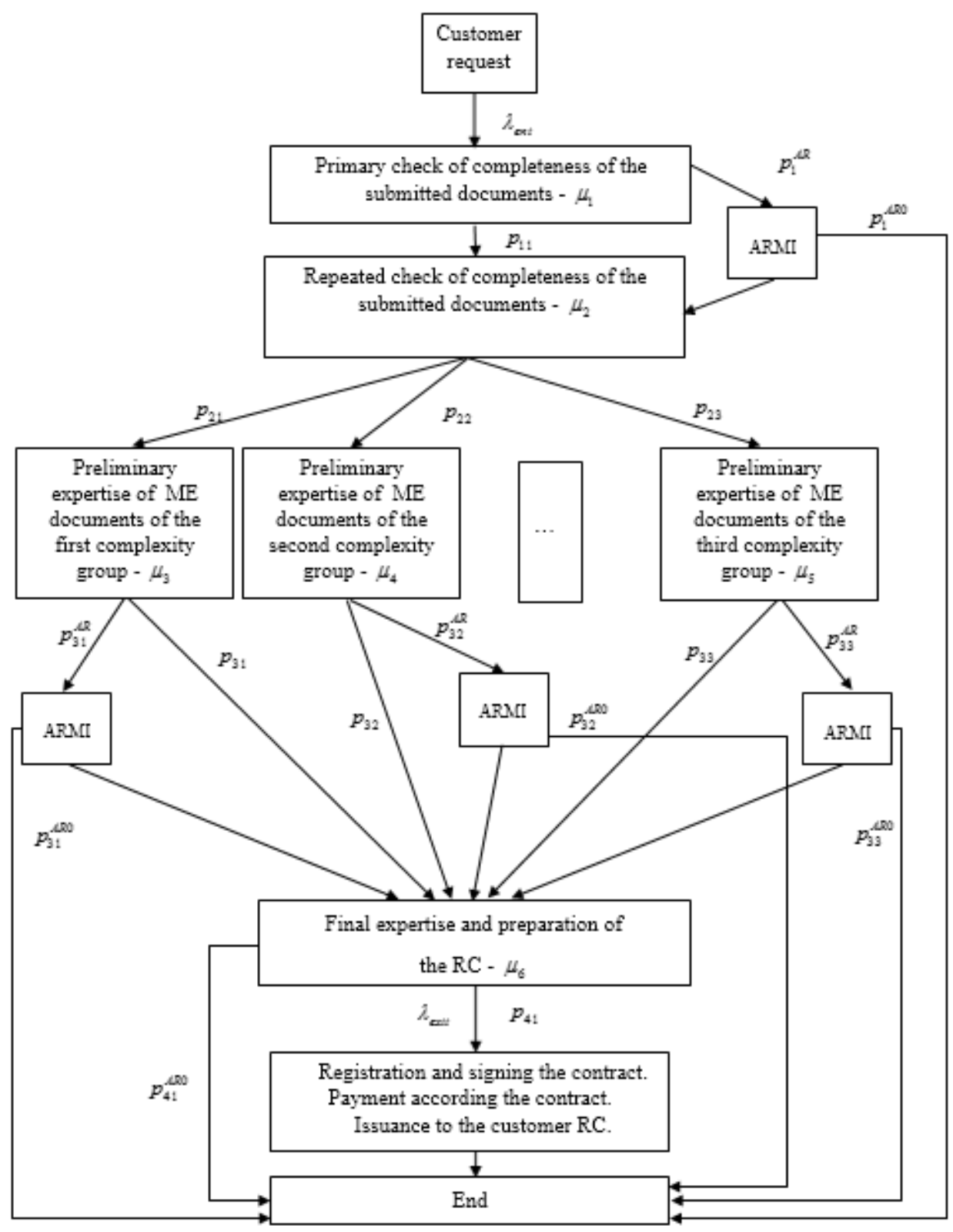

Fig. 1. Diagram of the multiphase queueing system. 


\section{The results of calculations}

Below are the results of calculations of multiphase queueing system performance containing $k=6$ single-phase systems. Incoming applications can be classified into one of three groups of complexity. The duration of time when the customer prepare and provide ARMI is not taken into account.

The calculations were carried out at the following values of the initial data: $\lambda_{\text {ent }}=8$ units $/$ month $, p_{11}=0,05, p_{1}^{A R}=0,95, p_{1}^{A R 0}=0,10, p_{21}=0,5, p_{22}=0,4, p_{23}=0,1$, $p_{31}=0,65, \quad p_{32}=0,65, \quad p_{33}=0,65, \quad p_{31}^{A R}=0,35, \quad p_{32}^{A R}=0,35, \quad p_{33}^{A R}=0,35, \quad p_{31}^{A R 0}=0,05$, $p_{32}^{A R 0}=0,05, p_{33}^{A R 0}=0,05, p_{41}=1, p_{41}^{A R 0}=0$.

The intensity of the serviced flow leaving the multiphase queueing system $\lambda_{\text {exit }}=7,11$ [units / month]. "Loss due to retirement of applications" due to the fault of the customer is about $11 \%$. Calculations were performed for different values of the total throughput of all single-phase queueing systems that vary in the range

$$
\sum_{i=1}^{6} \mu_{i}=60-96 \text { units / month . }
$$

Calculations have shown that the average time spent in the system and in the queue depends significantly on the distribution of this total throughput value for single-phase queueing systems. Calculations showed that the smallest value of the average time for stay an application in the queueing system is achieved if the throughput of each queueing system is proportional to the intensities of the flows passing through them.

Calculations showed that the time spent in the queue for the total service intensity 60 units / month, 72 units / month , 80 units / month , 96 units / month are respectively 49\%, $41 \%, 35 \%$ and $31 \%$ of the total time of the application in the system. At the same time, as the total service intensity increases, the time spent on the queue in the queue is reduced by 1,6 times, and the time spent in the system reduced by 2,2 times.

We estimate the required number of employees, which is able to provide the total capacity of a multiphase queueing system at all stages of work

$$
\sum_{i=1}^{6} \lambda_{i}=60 \text { units / month }
$$

and to ensure the maintenance of the incoming order flow with intensity $\lambda=8$ units / month.

Optimal distribution of the total throughput capacity for single-channel queueing systems (by stages of examination):

$$
\mu_{1}^{o p t}=16,22, \mu_{2}^{o p t}=14,68, \mu_{3}^{o p t}=7,34, \mu_{4}^{o p t}=5,87, \mu_{5}^{o p t}=1,47, \mu_{6}^{o n m}=14,42 .
$$

Here all quantities have the dimension of [units/month].

Let the average performance of one employee at different stages of the examination is respectively:

$$
\mu_{1}^{0}=200, \mu_{2}^{0}=30, \mu_{3}^{0}=10, \mu_{4}^{0}=6, \mu_{5}^{0}=2, \mu_{6}^{0}=20 .
$$

Here all quantities have the dimension of [units/month]. Then for maintenance of applications at various stages of the examination will be required

$$
n_{i} \approx \mu_{i}^{o p t} / \mu_{i}^{0}, i=1,2, \ldots, 6
$$

scientifis - technical worker. Thus, it takes

$$
\left[\sum_{i=1}^{6} n_{i}\right] \approx[3,74]=4
$$

scientifis - technical worker.

Thus, for a given incoming flow with intensity $\lambda=8$ units / month must be brought to the working of four professional scientific - technical workers. At the same time, the "net 
time" of servicing one application will not exceed $0.169 * 30=5.07$ days, including waiting time for the application to queue $-0.083 * 30=2.49$ days. Here, the time of receipt requested from the customer ARMI was not took into account.

Calculations have shown that the ratio of the labor cost during the expertise, which has a third level of complexity, to the labor cost of the expertise, which has the first level of complexity, is 3.12. The obtained result is in good agreement with the results obtained in [1].

In case of free entering streams (not simples Poison streams) the considered problem could be solved by means of methods of imitation simulation modelling $[5,6]$.

\section{Conclusion}

The mathematical model and software presented in the article makes it possible to implement a more differentiated approach to determining the labor costs of scientific and technical services for the expertise in comparison with the method of expert assessments [1] by including a larger number of complexity groups in the model. The stated approach can be used in the organization and planning of work on the expertise, and can be used as a basis method for calculating the real price of expertise.

The algorithms and approaches presented in the article can be effectively used in solving the problems of estimating the laboriousness of technological processes that take place in other systems whose activities are related to services and events that have the nature of a flow of random events.

\section{References}

1. T. E. Kuwik, A.A. Safonov, K.A.Tsybulnikova, Vestnik metrologa, 39 (2016)

2. A. I. Orlov, Expert estimations (Moscow, IDSTE Pub, 2002)

3. E. S. Ventzel, Operations Research (Moscow, Science Pub, 1972)

4. V.V. Grachev, A.N. Moiseev, A.A. Nazarov, V.Z. Yampolsky, Reports of TUSUR, 2, 26 (2012)

5. T. Altiok, B. Melamed, Simulation Modeling and Analysis with ARENA

(Amsterdam, Boston, Academic Press, 2007)

6. W.D. Kelton, R.P. Sadowski, D.A. Sadowski, Simulation with ARENA (McGraw Hill, Boston, 2002) 